DOI 10.35381/cm.v6i11.348

\title{
Ecologística: Estrategia basada en las 5 Rs para la satisfacción de necesidades
}

\section{Ecologistics: Strategy based on the 5rs for the fulfillment of needs}

\author{
Elvia Posada-Joven \\ eposada@urbe.edu.ve \\ Universidad Privada Dr. Rafael Belloso Chacín, Zulia \\ Venezuela \\ https://orcid.org/0000-0002-5968-4393
}

Recibido: 08 de abril de 2020

Revisado: 10 de mayo de 2020

Aprobado: 1 de julio de 2020

Publicado: 17 de julio de 2020

\section{RESUMEN}

El objetivo de esta investigación fue analizar la ecologística como estrategia basada en las 5 Rs para la satisfacción de necesidades. La fundamentación teórica fue de Flavio (2018), Unesco (2017) Manjarres y Chirino (2020), Chirino (2018), Severiche \& Acevedo (2013), entre otros. La investigación fue documental, orientada a evaluar las estrategias empleadas por la ecologistica conocida también como logística inversa o logística verde, dentro de la cadena de suministro. El resultado del estudio arguye que la ecologística como estrategia basada en las 5 Rs para la satisfacción de necesidades, debe propender a diseñar e implementar un modelo logístico que coadyuve a impulsar una cadena de logística integral para el manejo de residuos orgánicos producidos en los sectores de bajos recursos, que permitan el mejoramiento socio económico en comunidades vulnerables.

Descriptores: Ecologista; estrategia de desarrollo; calidad de vida; necesidades básicas; aprovechamiento de recursos. (Palabras del Tesauro UNESCO). 
Elvia Posada-Joven

\begin{abstract}
The objective of this research was to analyze ecology as a strategy based on the $5 \mathrm{Rs}$ to satisfy needs. The theoretical foundation was by Flavio (2018), Unesco (2017) Manjarres and Chirino (2020), Chirino (2018), Severiche \& Acevedo (2013), among others. The research was documentary, aimed at evaluating the strategies used by ecology also known as reverse logistics or green logistics, within the supply chain. The result of the study argues that ecology as a strategy based on the 5Rs for the satisfaction of needs, should tend to design and implement a logistics model that will help promote a comprehensive logistics chain for the management of organic waste produced in lowincome sectors. resources that allow socio-economic improvement in vulnerable communities.
\end{abstract}

Descriptors: Ecologist; development strategies; quality of life; basic needs; resources development. (Words from the UNESCO Thesaurus).

\title{
INTRODUCCIÓN
}

Desde el comienzo de la civilización, los seres humanos, así como los grupos sociales han experimentado necesidades, que ha originado una lucha por la obtención de recursos para impedir la escasez de aquellos considerados como de primera necesidad. De ahí que, las comunidades se plantearan nuevas formas que solucionaran la problemática. En este sentido, las sociedades desarrollan de manera empírica formas para poder atacar la carencia de algunos productos y servicios indispensables para satisfacer sus elementales privaciones.

En otras palabras, con el progreso de los pueblos se marca un deslinde entre ellos, dada la imposibilidad de enfrentar la lucha contra la escasez, aflorando nuevos conceptos como: pobreza, desarrollo social, así como calidad de vida; puesto que, al no contar con poder adquisitivo o capacidad para resolver las situaciones urgentes que viven, e incluso al no propender a conductas sociales para aglomerarse con sus pares sociales, que permitan emprender acciones conjuntas dirigidas a solucionar los dilemas que les aquejan, aparejando el incremento de necesidades insatisfechas convertidas en el desmejoramiento del bienestar común y buen vivir de las comunidades; por tanto, esto se ubica en la base de la pirámide identificada como estratificación social. 
De acuerdo con lo planteado anteriormente, surge la necesidad de implementar estrategias que puedan coadyuvar a una adecuada calidad de vida, en procura de aquellas de carácter accesorio, tales como: el auto aprovisionamiento de gas doméstico para la preparación de alimentos. En esta instancia, vale la pena comentar el rol que juegan las 5 Rs para la ecologistica como estrategia para satisfacer las necesidades; puesto que, ésta tiene sus cimientos, en la ecología aplicada al sector de la logística, tal como lo señala Revista TotalSafePack (2016).

Por consiguiente, tanto la ecologistica o ecología aplicada a la logística y las 5 Rs se fundamentan en la utilización de materiales reciclados o naturales empleando los criterios de Reducir, Reparar, Recuperar, Reutilizar y Reciclar en el proceso de fabricación de productos de su distribución, o valiéndose del uso de tecnologías limpias o amigables con el medio ambiente, para disminuir la contaminación dentro de todo lo que considera el sector logístico.

De ahí que, estas estrategias permitan optimizar los recursos con que se cuenta a fin de mejorar considerablemente el uso de los materiales logísticos y dar respuesta a la sociedad; para lo cual, se debe emplear el conocimiento desarrollado en las diferentes materias del saber con el propósito de ofrecer propuestas de proyectos lógicos sustentables que garanticen una mejor calidad de vida.

Volviendo la mirada a lo anterior, el presente estudio procura analizar la ecologística como estrategia basada en las 5 Rs para la satisfacción de necesidades y con ello evitar la escasez como elemento común en los sectores vulnerables; por tanto, hay que atender la problemática basada en estrategias limpias en resonancia con medidas medio ambientales. 


\section{METODOLOGÍA}

La investigadora efectuó el abordaje de este estudio basado en un examen introspectivo (Guillermo Morone, 2013), para lo cual se utilizó el tipo de investigación documental. En este sentido, Arias (2012, p. 27) sostiene que la investigación documental es un proceso basado en la búsqueda, recuperación, análisis, crítica e interpretación de datos secundarios, es decir, los obtenidos y registrados por otros investigadores en fuentes documentales: impresas, audiovisuales o electrónicas. Como en toda investigación, el propósito de este diseño es el aporte de nuevos conocimientos.

Del mismo modo, Fontaines (2012, p.130) señala que la modalidad documental se orienta hacia "la recolección de datos mediante el manejo de documentos de diversa índole"; por consiguiente, se procedió a la búsqueda de información preliminar en fuentes bibliográficas, escritas y electrónicas referidas, para organizar y desarrollar la presente investigación.

\section{ANÁLISIS Y DISCUSIÓN}

\section{Ecologística}

Resulta relevante el abordaje del término, con el fin de evitar confusiones; puesto que según la Revista TotalSafePack (2016), la ecologística no es otra que la ecología aplicada al sector de la logística, de manera que el utilizar materiales reciclados en el proceso de fabricación de productos para la distribución, así como aplicar tecnologías amigables para evitar la contaminación.

Con base a las ideas anteriores, la ecologistica es conocida también como logística inversa o logística verde, es el término vinculado con la sostenibilidad, que cada vez recobra más interés; puesto que, representa una preocupación generalizada por el cuidado del planeta, empujando a la sociedad para la concientización de las personas en la realización de actividades limpias y en concordancia con un medio ambiente apto que permita mejor calidad de vida. 
Desde otra perspectiva, la ecologistica tratada desde sus cimientos como ecología aplicada al sector de la logística y cuyos criterios conllevan a la utilización de materiales reciclados o naturales en el proceso de fabricación de productos de su distribución o aplicando la tecnología, para minimizar la contaminación durante todo el proceso logístico.

Asimismo, Manjarres y Chirino (2020) comentan que la logística ejerce un rol estratégico en las funciones empresariales ya que estas están vinculadas a la cadena de suministros tanto de los clientes, proveedores, así como a los procesos de producción, para ulteriormente lograr la satisfacción de las necesidades, los cuales redundan en beneficios.

Continúan Manjarres et al. (2020) advirtiendo que la logística verde o ecologistica surge como respuesta a la preocupación mundial de proteger y resguardar al medio ambiente; puesto que, el uso excesivo de recursos requiere que el sector empresarial debe propender a desarrollar estrategias para que sus procesos productivos se realicen con bajo un ecosistema amigable con el medio ambiente para minimizar el impacto ambiental que causa la producción de los productos a la sociedad. De manera que existen estrategias aplicables con la logística verde, tal como se ilustra en la próxima figura.

PROCESO LOGISTICA VERDE

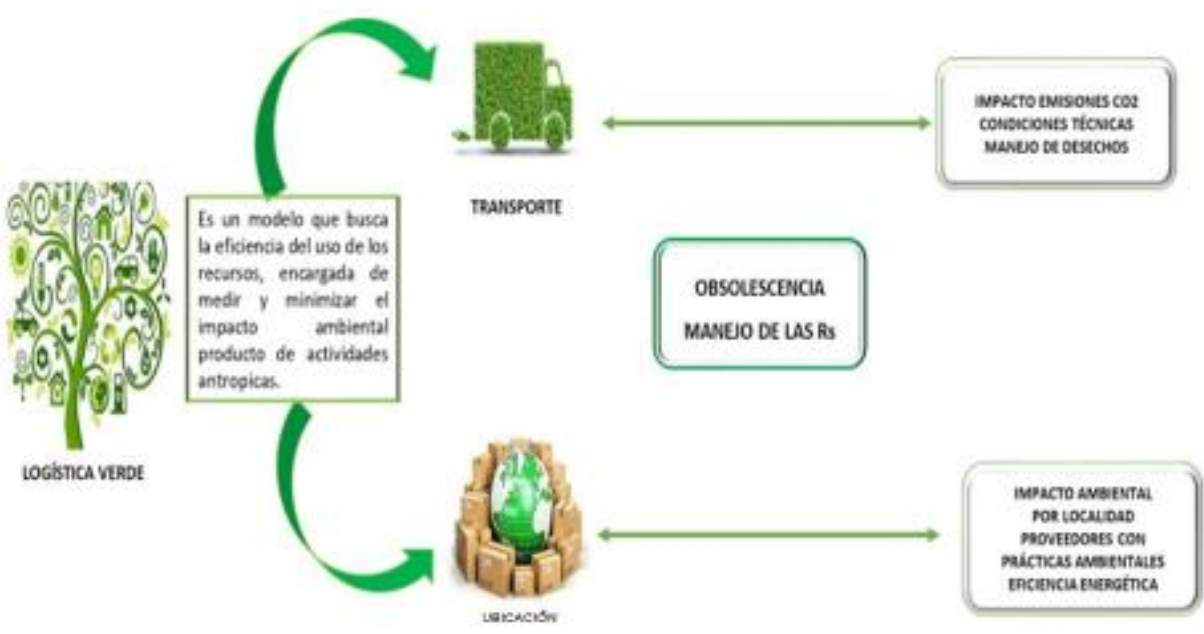




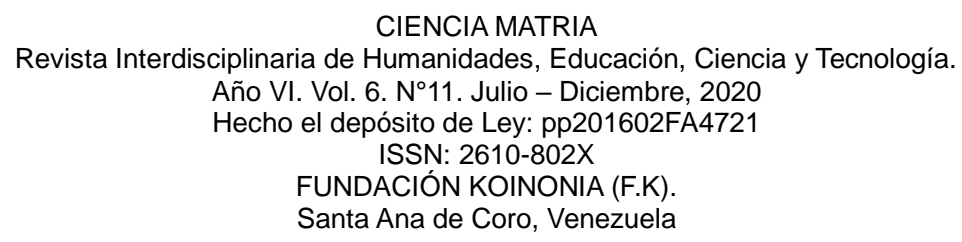

Elvia Posada-Joven

Por su parte, Chirino García (2018), advierte que la logística verde y la inversa, tienen distinciones donde la primera se enfoca en el consumo de los recursos naturales no renovables, emisión de contaminantes, utilización de vías, contaminación sonora y deposición de residuos, entre otros, mientras que la segunda considera los aspectos ambientales de todos los procesos logísticos y se enfoca más en la logística directa, pero basándose en una logística circular; ello es, rediseñar, reparar, reducir, reciclar, reutilizar o reventa.

Desde otro punto de vista, UNIGIS (2019) señala que la logística inversa, también conocida como el proceso de planificar, implementar y controlar el flujo de productos desde el punto de entrega hasta el punto de partida original de la forma más eficiente, eficaz y económicamente posible con el propósito de recuperar, aprovechar y disponer de ellos de manera adecuada.

En el mismo orden de ideas, el concepto aplica para la gran mayoría de los sectores, industrias, ya sean electrónicos, metales, alimentos empaquetados, transportes, entre otros. Sin embargo, existen industrias como la química, ganadera, pesquera, que implican un mayor control de su logística, puesto que la devolución significaría una pérdida total. Es decir, que la ecologistica contempla la medición además de desarrollo de estrategias, que reducen el impacto ambiental dentro de un proceso o actividad logística.

Dentro de otro contexto, Flavio (2018) comenta que una plataforma de ecologística es cuantitativa y cualitativamente diferente de los operadores de logística ubicados en un condominio industrial o también llamados sistémicos, a través de la plataforma, el sistema de distribución de carga puede aprovechar un centro de almacenamiento, operaciones de consolidación o desconsolidación de carga, transbordo de carga, personalizar productos, inspecciones de calidad, unificación de carga, empaque, documentación o gestión de inventario. 


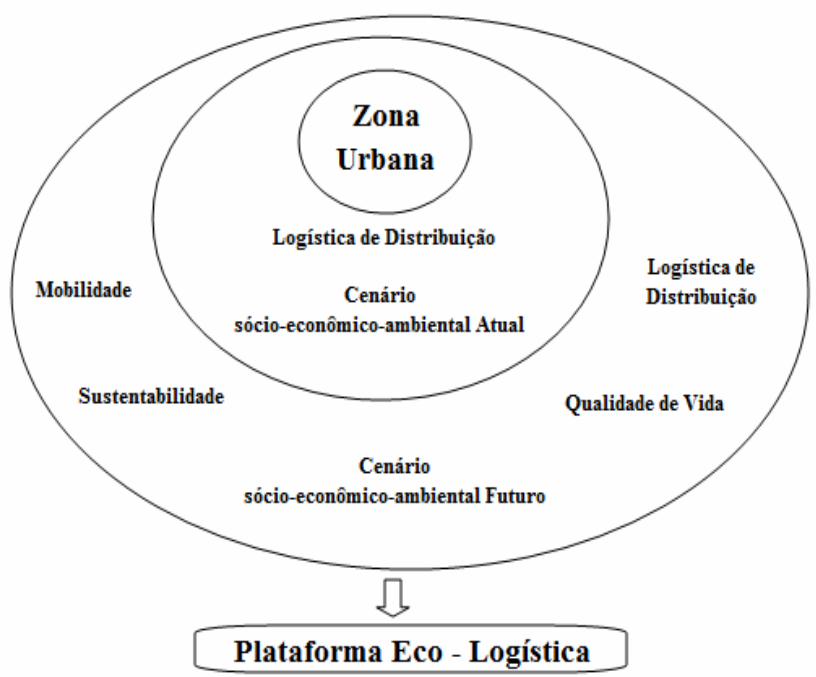

Fuente: Flavio (2018).

De acuerdo con las ideas anteriores, para la investigadora la ecologistica, tiene sus cimientos en la ecología además está sustentada en la logística inversa y en la economía circular, comprende un conjunto de actividades que van desde la recogida, desmontaje, desmembramiento ya sea de un producto o sus componentes que permitan el uso sostenible o utilización final; puesto que, ella está basada en la óptima utilización sin desmejorar el medio ambiente.

En virtud de las ideas planteadas anteriormente, la investigadora fija posición con lo planteado por Manjarres et al. (2020), quien realizan el abordaje sobre la ecologista de una manera amplia acotando que ésta está sustentada en la logística inversa y en la economía circular; puesto que, considera el uso de recursos que permitan el empleo sostenible de éstos; ya que, esta está basada en la disposición óptima sin desmejorar el medio ambiente. 


\section{Pirámide de necesidades}

En este segmento, se efectúa el abordaje de las necesidades humanas dentro del escenario mundial, en la sociedad se pueden observar ejemplos incuestionables en el devenir diario; puesto que la realidad es evidente; ya que, salta a la vista al pasearse por los centros urbanos en las calles principales de los mercados o en cualquiera de los sectores rurales, acompañada por un sin número de niños, mujeres y adultos en condiciones vulnerables; dicho de otra manera, en situación de pobreza, que permea hogares marcados por la imposibilidad de satisfacer las más elementales necesidades. En virtud de lo planteado anteriormente, las ciencias sociales han planteado una estratificación social comprendida a partir de la identificación de la satisfacción de necesidades; a su vez, alude un baremo de desarrollo social para los países; ello es, la segmentación de la población por grupos, separando a los pobres, de los no tan pobres, así como de los ricos. De manera que, otra forma de clasificación obedece a la segmentación de clase baja, media y alta; puesto que, es relativo a la estructura iconográfica de una pirámide, en la cual su base, con mayor capacidad está representada con la categoría de "pobres", en la media se ubican a los no tan pobres y en el reducido pináculo a los ricos.

Volviendo la mirada a lo planteado anteriormente, se evidencia como denominador común, las poblaciones ubicadas en la base de la pirámide, las cuales están en los estratos bajos relativos a la pobreza, como consecuencia de un resultado critico producido por el desmejoramiento en la calidad de vida, derivados de bajos ingresos percibidos. Asimismo, es el germen del disminuido desarrollo personal en cuanto al ámbito académico, laboral e inclusive cultural, que a decir de Busso, G. (2005), opina que las localidades alejadas de los centros económicos, con graves deficiencias en materia de servicios públicos, además de altos índices de desnutrición, informalidad comercial, explotación laboral, subempleo y desempleo, entre otros, constituyen un segmento de pasivo social en condiciones funestas. 


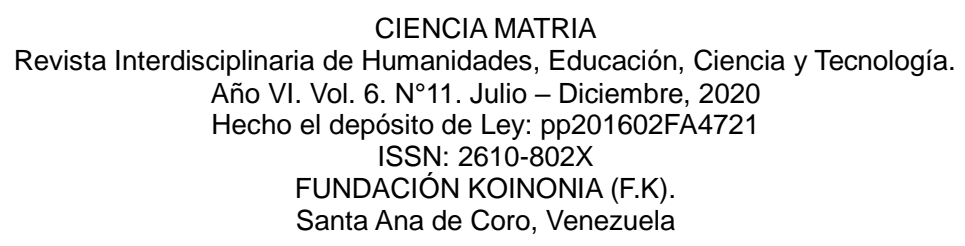

Elvia Posada-Joven

En efecto, lo antes mencionado podría ser considerado como el óbice para el desarrollo; por consiguiente, demanda atención prioritaria; en este sentido, la UNESCO (2017), en la agenda 2030 para el desarrollo sostenible, contempla dentro de los objetivos de esta, que la referida organización, pretende poner fin a la pobreza y el hambre, garantizando una vida sana, así como bienestar, para ello fomentar el crecimiento económico sostenido, inclusivo y sostenible además propender a la reducción de la contaminación y eliminar residuos en las fuentes de recursos.

Aunado a lo anterior, Busso, G. (2005) comenta que existen varias acepciones de pobreza, acotando que este sustantivo invoca situaciones de carencias de bienes, servicios materiales que permitan vivir y funcionar adecuadamente como miembro de la sociedad. De ahí que, para satisfacer las necesidades se deben desarrollar herramientas, así como la estructuración en red para ejercicios decisorios que incidan de manera positiva el entorno.

\section{Estrategias para la satisfacción de necesidades}

Cabe agregar, que una vez identificadas las características propias a los sectores que conforman la base de la pirámide, resulta menester establecer estrategias orientadas a emprender acciones para enfrentar tales flagelos, con el propósito de atacar sus causas y efectos mediante soluciones creativas e innovadoras a partir de una economía circular, optimizando los residuos, desechos para conformar capacidades destinadas a la creación de cadenas de valor desde y para las comunidades.

De acuerdo con lo mencionado anteriormente, el término pobreza, evoca un disminuido o inexistente poder adquisitivo, incapacidad para racionalizar el aprovechamiento de los recursos propios, para resolver problemáticas comunes, así como la desconexión social entre comunidades, que exacerba la carencia de servicios públicos, además del desarrollo personal y empresarial, entre otros aspectos.

Ahora bien, surge la posibilidad de crear un plan estratégico cuya propuesta de valor sea fomentar emprendimientos sostenibles y sustentables conformados por integrantes de 


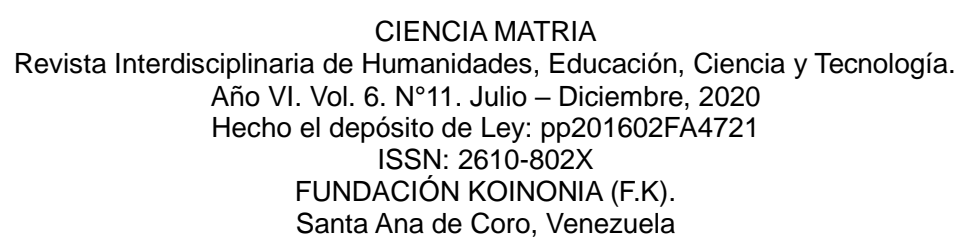

Elvia Posada-Joven

las mismas comunidades, que coadyuven a auto proveerse de gas doméstico, a partir, de un sistema de gestión de residuos orgánicos, mediante el uso de las 5 Rs. Esto significa que con estas estrategias, se busca reducir, reutilizar, reciclar los recursos y gastos de producción innecesaria para todo aquel material cuya naturaleza lo requiera, así como su composición permita recuperar lo dado por innecesario, desperdicio o basura, reciclándolo para conceder una nueva utilización, esto constituiría un milagro económico al significar una forma moderna del manejo de los recursos.

Lo anterior contempla tres elementos característicos de la pobreza; puesto que, impulsa la mecanización del aparato productivo, generaría nuevas fuentes de trabajo, impactando el poder adquisitivo en la comunidad; adicionalmente, refiere la solución de enormes problemas como lo son el acceso a gas doméstico en sectores vulnerables o de bajos recursos ya que no existen líneas directas o acceso a las viviendas; por lo que, deben pagar por la recarga de bombonas, lo que representa una dificultad, además de costosa debido a la distancia existente entre los centros económicos y la comunidad.

Por último, la modalidad innovadora propuesta, resuelve problemas de calidad de vida, salud, medio ambiente, entre otros, provocados por la recolección ineficiente de los desechos, desperdicios o basura doméstica. A los efectos de este, Rendón (2016), comenta que estos desechos, desperdicios o basura doméstica, es sumamente preocupante, ya que los problemas relativos a la contaminación y deterioro generalizado del medio ambiente son considerados, tanto o más apremiantes que los de aprovisionamiento de agua potable, alcantarillado, energía eléctrica, transporte, así como vías de comunicación, ya que la ausencia de un medio ambiente adecuado conduce a que los servicios mencionados se conviertan en actividades irrelevantes para una comunidad con problemas de supervivencia.

De manera que el abordaje de esta investigación, contempla varios de los objetivos. establecidos por la UNESCO (2017) en la agenda 2030 para el desarrollo sostenible, como lo son; erradicar la pobreza, hambre cero; salud y bienestar, energía asequible; no contaminante, trabajo decente y crecimiento económico; industria, innovación e 
infraestructura; ciudades y comunidades sostenibles, además; producción y consumo responsable, vida de ecosistemas terrestres; alianzas para lograr los objetivos, entre otros.

Resulta pertinente, la relación con los acuerdos derivados de la Conferencia de las Naciones Unidas sobre Medio Ambiente y Desarrollo sostenible en la Cumbre de Rio, tal como lo ratifican los autores antes citados. Desde otra perspectiva, lo comentado anteriormente se apoya en dos escenarios, uno relacionado con la recolección y clasificación de la materia orgánica; que invita a la conformación de cuadrillas sectoriales, conformadas por individuos de la comunidad, quienes se encarguen de recoger los desechos para posteriormente clasificarlos en atención a su naturaleza orgánica, separando los metales, plástico y vidrio, entre otros, del resto que será puesto a la orden de la fase siguiente en el proceso de producción del gas doméstico sostenible (Biogás). De ahí que, se podría utilizar y comercializar los primeros a la industria de envases, aplicando con ello una de las estrategias de las 5 Rs, como lo es la reutilización y el reciclaje. A lo anterior se agrega, la decisión de abordar el segundo escenario referido a los aspectos tecnológicos concernientes a la gestión bioquímica, la cual es necesaria para la transformación de la materia seca en gas doméstico; el cual contempla la utilización de biodigestores, a tales efectos; se observan dos posibilidades, siendo la primera instalar procesos particulares de forma casera y la segunda, establecer un centro de transformación y llenado central para toda la comunidad.

Finalmente, para lograr los objetivos fijados, se hace necesario observar la demanda vinculada con los medios de transporte, organización, clasificación y almacenamiento de los desechos sólidos; además la construcción de biodigestores para el proceso de conversión, así como el traslado del producto terminado generado hasta la disposición final, aprovechando los sedimentos heredados del proceso principal para transformarlo en abono líquido, todo lo cual configura la necesidad perentoria del establecimiento de una red ecologística. 


\section{Aspectos Técnicos de la estrategia}

En cuanto a este segmento, se puede inferir que la interacción humana ha aportado exceso de nutrientes orgánicos e inorgánicos producto del uso descontrolado de agroquímicos, además del manejo incorrecto de aguas residuales; por otro lado, la falta de conciencia ecológica de la población, así como el poco cumplimiento de las leyes ambientales por parte de las industrias que realizan actividades antrópicas, aunado al carente seguimiento de la normativa ambiental, han provocado un considerable pasivo ambiental.

Dentro de otro contexto, las labores de operación, mantenimiento, sostenibilidad, entre otras, garantizan un adecuado funcionamiento de los biodigestores. En las plantas construidas generalmente se presenta flotación de material sólido, el cual podrá ser tratado; puesto que, forma una capa gruesa obstaculizando la salida del efluente y deteriorando su calidad.

Asimismo, para la preservación ambiental se manejan criterios de bioenergías aplicadas a los sistemas productivos. Es evidente entonces, que el aspecto práctico resalta la estructuración de una planta productora de biogás, la cual funcionaría con materia prima proveniente de la recolección de afluentes encontrados en los vertederos, basándose con ello en la utilización de la economía circular o estrategia logística de las 5Rs. Lo anterior, es ratificado por varios autores, tal como lo sostiene Castro (2016), quien comenta que el biogás puede ser generado a partir de residuos orgánicos, lo cual constituye una fuente de energía sostenible y renovable que coadyuva al ahorro de energía y reduce los daños ambientales.

De acuerdo con las ideas planteadas anteriormente, el diseño y puesta en marcha de una planta de generación en base a biogás, representa una alternativa viable, basada en el uso e incorporación de tecnologías limpias cuyo impacto ambiental, así como el manejo de residuos representa una mitigación eficiente al tratamiento de la contaminación producto de emisiones de gases de efecto invernadero, las cuales deteriorarían el ecosistema. 


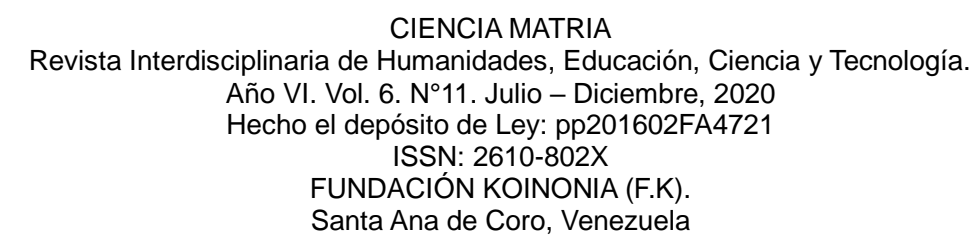

Elvia Posada-Joven

En resumen, para atender la problemática ambiental, económica y social, resulta urgente el diseño y la puesta en ejecución de un nuevo ecosistema, el cual requerirá del apoyo económico del Estado; puesto que, demanda una inversión para cubrir las necesidades colectivas a partir del tratamiento de efluentes con lo cual se mejorará la calidad de vida de la sociedad y además el uso de tecnologías limpias eliminaría el impacto negativo en el medio ambiente.

\section{DISCUSIÓN}

Al contrastar lo enunciado en la Revista TotalSafePack (2016), que advierten los cimientos que concederá el surgimiento de la logística; puesto que, la ecologistica se fundamenta en la ecología; por lo tanto, se convierte en pilar relevante; dado que utiliza materiales reciclados dentro de los procesos de fabricación de los productos, para luego distribuirlos; además, de la aplicación de tecnologías limpias que no afectan al medio ambiente y reducen la contaminación. A lo anterior, se agrega, la postura de Favio (2018) al incorporar su argumentación cuali-cuantitativa sobre el manejo logístico fundamentado en la reducción de la contaminación medio ambiental.

En el mismo orden de ideas, UNIGIS (2019), comparte la postura explicada anteriormente ya que sostiene que la logística observada desde esa perspectiva no es más que la invocación de la logística verde o inversa, la cual apareja un proceso de planificación, implementación y control del flujo de productos, el cual comprende varias fases que van desde el inicio hasta la entrega; pero llevados de forma eficiente, eficaz, efectiva y económicamente posible que posibilite la recuperación, aprovechamiento, así como la disposición de los elementos de manera adecuada.

Dentro de otro contexto, al cotejar la postura de Manjarres y Chirino (2020) logística verde o ecologistica, se evidencian coincidencias con lo planteado por Chirino García (2018), pues concurren en la opinión sobre la utilización en los procesos productivos de recursos que protejan el medio ambiente, además de evitar las actividades antrópicas; en otras palabras, operaciones efectuadas bajo un ecosistema amigable con el medio ambiente 


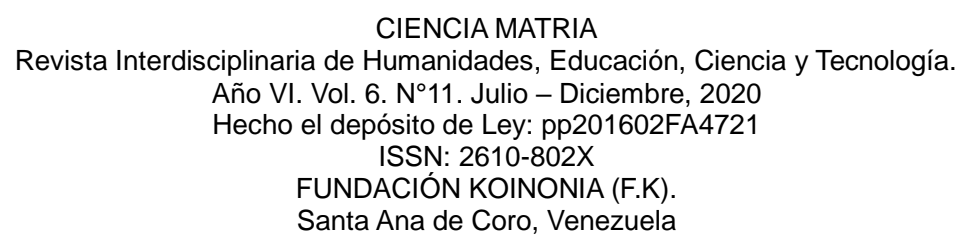

Elvia Posada-Joven

para minimizar el impacto ambiental, colocándose en resonancia con los objetivos del desarrollo sostenible contemplados en la agenda 2030 emanada de la UNESCO. Con respecto a la pirámide de necesidades, el fundamento de esta radica en la pobreza; a los efectos de este, Busso (2005), sostiene que el término está vinculado a las carencias de bienes o servicios que permitan la pervivencia y funcionamiento de los integrantes de una sociedad. Por consiguiente, resulta urgente y necesario satisfacer esas necesidades de bienes o servicio, implementando acciones que incidan positivamente en una comunidad y su entorno.

Es por ello que, la UNESCO (2017) en la Agenda 2030 para el Desarrollo Sostenible, contempla los objetivos 1, 10 y 11 que enuncian la erradicación de la pobreza, reducir las desigualdades promoviendo el crecimiento económico sostenido, inclusivo y reduciendo la contaminación mediante el aprovechamiento de los residuos en las fuentes de recursos. En esta instancia, la investigadora fija posición con lo establecido en la agenda 2030 de la UNESCO (2017), dado que la misma establece con precisión los objetivos para la atención de las necesidades básicas de la sociedad, pues pretende poner fin a la pobreza aunado a atacar las desigualdades auspiciando un crecimiento económico con criterios de sostenibilidad.

Tal como se observa, ha llegado el momento de efectuar el aporte a la comunidad científica como resultado de esta investigación. En este sentido, para lograr estar en resonancia con los objetivos establecidos en la agenda 2030 de Desarrollo sostenible de la UNESCO (2017), resulta perentorio el tratamiento de los desechos que incluya la planificación, organización, clasificación y control de residuos orgánicos a fin de poder diseñar e implementar una planta de biogás que posibilita valorización de los residuos para uso energético.

Ahora bien, una vez realizado el proceso de tratamiento de los desperdicios orgánicos, se pueden utilizar también como abono para la tierra estando a la par de las estrategias relacionadas con las $5 \mathrm{Rs}$, donde se aparejaran beneficios; puesto que, reducen la contaminación además de resolver el problema de generación de gases con efecto 
invernadero, todo lo cual representan una amenaza para la calidad de vida y pervivencia futura de las nuevas generaciones.

\section{REFLEXIONES FINALES}

Al analizar la ecologística como estrategia basada en las 5 Rs para la satisfacción de necesidades, la investigadora concluye que, dado que el biogás es una fuente de energía renovable, así como tecnología limpia amigable con el medio ambiente, el uso de este ecosistema propenderá a utilizar el combustible fósil como medio para la generación energética, lo cual mejorará las condiciones ambientales producto de la contaminación proveniente del quemado de éste. Asimismo, partiendo de la recolección y tratamiento de desechos se implementaría la utilización de las estrategias de las 5 Rs en concordancia con algunos de los objetivos establecidos en la agenda 2030 de la UNESCO con el fin de proporcionar un mejor vivir a las comunidades.

Por otra parte, la investigadora asiente que se observaría un adecuado proceso de producción en las industrias, las cuales mitigarían las actividades antrópicas. A lo anterior se añade que, la planta productora de biogás puede realizar mediante el uso de tecnologías limpias, la transformación de efluentes residuales e industriales a energía, coadyuvando a solucionar la problemática de déficit energético en las comunidades más vulnerables.

\section{REFERENCIAS CONSULTADAS}

Busso, G. (2005). Pobreza, exclusión y vulnerabilidad social. Usos, limitaciones y potencialidades para el diseño de políticas de desarrollo y de población.[ Poverty, exclusion and social vulnerability. Uses, limitations and potentialities for the design of development and population policies], 1. (Disponible en: https://n9.cl/tzyko. Consultado el 10/06/2020).

Cabeza, M. y Reimi, M. (2005). Una aproximación metodológica al problema de los residuos de envase y embalajes. [A methodological approach to the problem of packaging waste]. Ciencia e Ingeniería, vol. 26, núm. 1, Universidad de los Andes. Mérida-Venezuela. 
Castro S, A. (2016). Beneficios Sociales, Ambientales y Económicos en la implementación de plantas de Biogás para el tratamiento de desechos ganaderos en Chile.[ Social, Environmental and Economic Benefits in the implementation of Biogas plants for the treatment of livestock waste in Chile].(Disponible en: https://n9.cl/yu8k. Consultado el 10/06/2020).

Chirino, R (2018). Logística verde y Gestión ambiental: Desafío para el gobierno corporativo en las organizaciones lucrativas. [Green Logistics and Environmental Management: Challenge for corporate governance in profit organizations]. IJRDO Journal of Social Science and Humanities Research. Volume-3, Issue-5, May, 2018. (Disponible en: https://n9.cl/zvb3. Consultada el 08/06/20).

Da Silva, C. Rodriguês, R. , et al. (2018) "A Ecologistica e o uso correcto de Navios e Conteinëres como fator de desenvolvimiento sustentável e de competitividad empresarial: Um estudio de caso Na empresa Hamburg Süd. " Revista Gestão \& Sustentabilidade Ambiental 7.1, pág.: 79-99. Disponible en: https://n9.cl/3t5ep. Consultado el 08/06/2020).

Dekker, R., Bloemhof, J., \& Mallidis, I. (2011). Operations Research for green logistics Anover view of aspects, issues, contributions and challenges. European Journal of Operational Research, 671 - 679. Europa. (Traducción libre Chirino 2020).

Embum, X., Zuleta, A. (2001). Sistemas de Gestión Medioambiental. [Environmental Management Systems]. Editorial Colex, Madrid-España.

Fontaines, T. (2012). Metodología de la investigación. Pasos para realizar el Proyecto de investigación. [Investigation methodology. Steps to carry out the research project]. Caracas Venezuela. Júpiter editors.

Gómez M., Rodrigo A.; Correa E., Alexnader A. y Vásquez H., Laura S. (2012). Logística inversa, un enfoque con responsabilidad social empresarial. [Reverse logistics, an approach with corporate social responsibility]. Criterio Libre, 10 (16), Pp. 143-158. Disponible en: https://n9.cl/6q8k. Consultado el 10/06/2020).

Hernández y otros (2016). Metodología de la investigación. [Investigation methodology]. Sexta edición. Editorial Mc Graw Hill. México. D.F. 
Londoño, D. (2017). Factibilidad para la creación de una empresa de recuperación de metales y materiales reciclables en la ciudad de Pereira. [Feasibility for the creation of a company for the recovery of metals and recyclable materials in the city of Pereira]. Proyecto de grado requerido por el Programa de Negocios Internacionales de la Universidad Católica de Pereira.

Manjarres, A y Chirino, R. (2020). Logística verde. Reto Gerencial para el manejo de la Gestión Ambiental Sostenible. [Green logistics. Management Challenge for the management of Sustainable Environmental Management]. (Disponible en https://n9.cl/wjw2i. Consultada 08/06/2020).

Numata, F. (2020). Plataforma Eco-logistica como estrategia para reduçao do impacto urbano. (Disponible en: https://n9.cl/oevz. Consultado el 09 de junio de 2020).

Robinson, Norma (2004). Logística verde: estrategias para imprentarla y casos exitosos en América Latina.[ Green logistics: strategies to print it and successful cases in Latin America]. Zona logística. Bogotá.

Rondón, E., Narea, M. Pacheco, J., Contreras, E. y Gálvez, A. (2016). Guía general para la gestión de residuos sólidos domiciliarios.[ General guide for the management of household solid waste]. Publicación de las Naciones Unidas.

Serra de La Figuera, D. (2005). La logística empresarial en el nuevo milenio. [Business logistics in the new millennium]. Barcelona: Gestion 2000.

Severiche, S., C., \& Acevedo, B., R. (2013). Biogás a partir de residuos orgánicos y su apuesta como combustibles de segunda generación.[ Biogas from organic waste and its commitment as second generation fuels]. Ingenium] Revista de la facultad de ingeniería, 14(28), 6-15. (Disponible en: https://n9.cl/0axm. Consultado el 10/06/2020).

Taulois, T y Araujó, M. (2015). Rede 5Rs: A base Estrategica para a operacionalização da política nacional de residuos solidos, no ámbito industrial. (Disponible en: https://n9.cl/1h68. Consultado el 08/06/2020).

TotalsafePack (2017). (Disponible en https://n9.cl/0o5t. Consultada 08/06/2020). 
Elvia Posada-Joven

UNESCO (2017). Agenda 2030 para el desarrollo sostenible. [2030 Agenda for sustainable development]. (Disponible en https://n9.cl/dhxs. Consultada el 10/06/2020).

Unigis (2019). (Disponible en: https://n9.cl/j0t6. Consultada el 08/06/20).

Zúñiga Pérez, Á. S. (2010). Evaluación Técnico Económica y Diseño de una Planta Generadora de Electricidad a Partir del Biogás.[ Technical Economic Evaluation and Design of an Electricity Generating Plant from Biogas]. (Disponible en: https://n9.cl/ymp3. Consultada el 10/06/2020). 